\title{
MEAN EXIT TIME FROM A BUMPY SPHERE
}

\author{
MARK A. PINSKY
}

(Communicated by Lawrence Gray)

ABstract. We find the solution of $\Delta f=-1$ in a deformation of a sphere in $\mathfrak{R}^{d}$.

If $D$ is a bounded region in $\mathbf{R}^{d}$, the solution of the Poisson equation $\Delta u=$ -1 satisfying the boundary condition $u=0$ on $\partial D$ can be identified as the mean exit time of the Brownian motion process $\left\{X_{t}, t \geq 0\right\}$. This can be seen by appealing to the Dynkin formula [1, Theorem 5.1, p. 145], which states that for any $C^{2}$ function $f$ and any stopping time $T$ with finite expectation, $E_{x}\left[f\left(X_{T}\right)\right]-f(x)=E_{x}\left\{\int_{0}^{T} \Delta f\left(X_{s}\right) d s\right\}$. Taking $f=u$ and $T=\inf \{t: t>$ $\left.0, X_{t} \notin D\right\}$ gives the required identification $u(x)=E_{x}[T]$. In the remainder of this paper the results are stated and proved in terms of the function $u$, with no explicit reference to Brownian motion.

Let $D_{\varepsilon, R}$ be the $d$-dimensional region defined by the inequality

$$
D_{\varepsilon, R}:=\left\{\left(x_{1}, \ldots, x_{d}\right): \sqrt{x_{1}^{2}+\cdots+x_{d}^{2}}<R(1+\varepsilon g(x /|x|))\right\}
$$

where $\varepsilon, R>0$ and $g: S^{d-1} \rightarrow \mathfrak{R}$ is a smooth real-valued function. If $g$ is not identically zero and $\varepsilon<\frac{1}{\sup |g|}$, the region $D_{\varepsilon, R}$ is smooth with a boundary $\partial D_{\varepsilon, R}$ which is diffeomorphic to the standard sphere $S^{d-1}$. We consider the boundary value problem

$$
\begin{array}{cl}
\Delta u_{\varepsilon}=-1 & \text { in } D_{\varepsilon, R}, \\
u_{\varepsilon}=0 & \text { on } \partial D_{\varepsilon, R} .
\end{array}
$$

We will prove the following proposition, where we adopt the convention that $1 / 0=\infty$.

Proposition. For $0 \leq \varepsilon<\left[\sup _{|x|=1}|g(x)|\right]^{-1}$ we have

$$
u_{\varepsilon}(0)=\frac{R^{2}}{2 d}+\varepsilon \frac{R^{2}}{d} \int g(\theta) d \theta+\frac{\varepsilon^{2} R^{2}}{2 d} \int g(\theta)^{2} d \theta,
$$

Received by the editors July 28, 1992 and, in revised form, February 8, 1993.

1991 Mathematics Subject Classification. Primary 60J60.

Key words and phrases. Mean exit time.

Research supported by ARO Grant 28905-MA. 
where the integrals are over the unit sphere with respect to normalized surface measure.

Corollary 1. If $u_{\varepsilon}(0)=R^{2} / 2 d+o(\varepsilon)$, when $\varepsilon \searrow 0$, then $\int g(\theta) d \theta=0$; i.e., the bumps average to zero.

Corollary 2. If $u_{\varepsilon}(0)=R^{2} / 2 d+o\left(\varepsilon^{2}\right)$ when $\varepsilon \searrow 0$ then $g(\theta) \equiv 0$; i.e., $D_{\varepsilon, R}$ is a round sphere of radius $R$.

Proofs. We transform the problem to the standard sphere $D_{0, R}$ by the transformation $x \rightarrow y$ defined by $\rho=r /[1+\varepsilon g(\theta)]$ where the notation is chosen so that $r=\sqrt{x_{1}^{2}+\cdots+x_{d}^{2}}, \rho=\sqrt{y_{1}^{2}+\cdots+y_{d}^{2}}$, and $\theta=x /|x|=y /|y|$.

The indicated transformation is nondifferentiable at the origin unless $g$ is a constant. Away from the origin we can compute the partial derivatives as

$$
u_{r}=\frac{u_{\rho}}{1+\varepsilon g(\theta)}, \quad u_{r r}=\frac{u_{\rho \rho}}{(1+\varepsilon g(\theta))^{2}} .
$$

The Laplacian is transformed as

$$
\begin{aligned}
\Delta u & =u_{r r}+\frac{(d-1)}{r} u_{r}+\frac{1}{r^{2}} \Delta^{S^{d-1}} u \\
& =\frac{1}{(1+\varepsilon g(\theta))^{2}}\left[u_{\rho \rho}+\frac{(d-1)}{\rho} u_{\rho}+\frac{1}{\rho^{2}} \Delta^{S^{d-1}} u\right] .
\end{aligned}
$$

The new differential equation is $\Delta u=-(1+\varepsilon g(\theta))^{2}, 0<\rho<R$. We look for the solution in the form $u_{\varepsilon}=u_{0}+\varepsilon u_{1}+\varepsilon^{2} u_{2}$. This can happen for all $\varepsilon$ iff the following auxiliary conditions are satisfied:

$$
\begin{array}{lll}
\Delta u_{0}=-1, & 0<\rho<R, \theta \in S^{d-1}, & u_{0}(R, \theta)=0 \\
\Delta u_{1}=-2 g(\theta), & 0<\rho<R, \theta \in S^{d-1}, & u_{1}(R, \theta)=0 \\
\Delta u_{2}=-g(\theta)^{2}, & 0<\rho<R, \theta \in S^{d-1}, & u_{2}(R, \theta)=0 .
\end{array}
$$

The first equation has the explicit solution $u_{0}=\left(R^{2}-\rho^{2}\right) / 2 d$.

The second and third equations are solved by means of the Green function $G\left(\rho, \theta ; \rho^{\prime}, \theta^{\prime}\right)$ as $u_{1}(\rho, \theta)=\int G\left(\rho, \theta ; R, \theta^{\prime}\right)\left[-2 g\left(\theta^{\prime}\right)\right] d \theta^{\prime}$ with a similar formula for $u_{2}\left[3\right.$, Chapter 8]. At the center we have $G\left(0, \theta ; R, \theta^{\prime}\right)=$ const, independent of $\theta, \theta^{\prime}$. The normalization is obtained by referring to the case $g(\theta)=1$. From these considerations we obtain the indicated values for $u_{1}(0)$ and $u_{2}(0)$, completing the proof of the proposition. The corollaries follow immediately from the proposition.

It should be noted that we have not simply proved an asymptotic formula for the solution but have obtained an exact formula. The identity of the stated formula with the exact solution follows from the uniqueness of solutions of the equation $\Delta u=-1$ and the theorem on removable singularities of elliptic equations [2]; indeed, by defining $u_{0}, u_{1}, u_{2}$ as above, the function $u_{0}+\varepsilon u_{1}+$ $\varepsilon^{2} u_{2}$ is a bounded solution of the equation in a deleted sphere, and hence equal to the true solution.

\section{ACKNOWLEDGMENT}

We thank Leon Karp for a helpful conversation. 


\section{REFERENCES}

1. A. Friedman, Stochastic differential equations and applications, Vol. 1, Academic Press, New York, 1975.

2. D. Gilbarg and J. Serrin, On isolated singularities of solutions of second order elliptic equations, J. Analyse Math. 4 (1954-56), 309-336.

3. M. Pinsky, Partial differential equations and boundary value problems with applications, second ed., McGraw-Hill, New York, 1991.

Department of Mathematics, Northwestern University, Evanston, Illinois 602082730

E-mail address: pinsky@math.nwu.edu 